\title{
Hadron production in hot and dense nuclear matter
}

\section{A. Lavagno*}

Dipartimento di Fisica, Politecnico di Torino and INFN, Sezione di Torino, Italy

E-mail: andrea.lavagno@polito.it

\begin{abstract}
We study the hadron production at finite values of temperature and baryon density by means of an effective relativistic mean-field model with the inclusion of the full octet of baryons, the $\Delta$-isobar degrees of freedom and the lightest pseudoscalar and vector mesons. These last particles are considered in the so-called one-body contribution, taking into account of an effective chemical potential and mass depending on the self-consistent interaction between baryons. The analysis is performed by requiring the Gibbs conditions on the global conservation of baryon number, electric charge fraction and zero net strangeness. In this context, we study the behavior of different particle-antiparticle ratios and strangeness production.
\end{abstract}

The 2011 Europhysics Conference on High Energy Physics, EPS-HEP 2011,

July 21-27, 2011

Grenoble, Rhône-Alpes, France

${ }^{*}$ Speaker. 
We study the hadronic equation of state (EOS) by requiring the global conservation of baryon number, electric charge fraction and zero net strangeness in the range of finite temperature and density. The study is performed by means of an effective relativistic mean field model with the inclusion of the octet of the lightest baryons, the $\Delta$-isobar degrees of freedom and the lightest pseudoscalar and vector mesons [1, 2, 3]. These last particles have been considered in the so-called one-body contribution, taking into account their effective chemical potentials depending on the self-consistent interaction between baryons [4]. In analogy to the effective meson chemical potentials, the effective meson masses are expressed as a difference of the effective baryon masses respecting the strong interaction and the main processes of the meson production/absorption involving different baryons. Such assumption implies a mechanics in which the vacuum meson masses are reduced (enhanced) if the ratio between the $\sigma$-meson field coupling with the heavier baryon ( $\Delta$ or hyperon particles involved in the meson production/absorption) and the nucleon one, is greater (lower) than one. A variation of the effective meson masses in-medium simulates, in our simple scheme, the relevance of meson-meson and meson-baryon self-interaction in the nuclear medium at finite temperatures and baryon densities.

The chemical potential of particle of index $i, \mu_{i}$, can be expressed in term of the three independent chemical potentials: $\mu_{i}=b_{i} \mu_{B}+c_{i} \mu_{C}+s_{i} \mu_{S}$, where $b_{i}, c_{i}$ and $s_{i}$ are, respectively, the baryon, the electric charge and the strangeness quantum numbers of the $i$-th hadronic species. At low baryon density and high temperature, the contribution of the lightest pseudoscalar and vector mesons to the total thermodynamical potential becomes very relevant. From a phenomenological point of view, we can take into account of these contributions by incorporating such mesons by adding to the thermodynamical potential their one-body contribution, i.e. the contribution of an ideal Bose gas with an effective chemical potential. Following Ref. [4], the values of the meson effective chemical potentials are fixed from the "bare" chemical potentials and writing them in terms of the corresponding baryon effective chemical potentials, respecting the strong interaction. For example, for pions (and rho mesons) we have that $\mu_{\pi^{+}}=\mu_{\rho^{+}}=\mu_{C} \equiv \mu_{p}-\mu_{n}$ and its effective chemical potential can be written as

$$
\mu_{\pi^{+}}^{*}=\mu_{\rho^{+}}^{*} \equiv \mu_{p}^{*}-\mu_{n}^{*}
$$

For the other mesons, we have

$$
\begin{aligned}
& \mu_{K^{+}}^{*}=\mu_{K^{*+}}^{*} \equiv \mu_{p}^{*}-\mu_{\Lambda\left(\Sigma^{0}\right)}^{*}, \\
& \mu_{K^{0}}^{*}=\mu_{K^{* 0}}^{*} \equiv \mu_{n}^{*}-\mu_{\Lambda\left(\Sigma^{0}\right)}^{*},
\end{aligned}
$$

while the others non-strange neutral mesons have a vanishing chemical potential. Thus, the effective meson chemical potentials are coupled with the meson fields related to the interaction between baryons. This assumption represents a crucial feature in the EOS at finite density and temperature and can be seen somehow in analogy with the hadron resonance gas within the excluded-volume approximation. There the hadronic system is still regarded as an ideal gas but in the volume reduced by the volume occupied by constituents (usually assumed as a phenomenological model parameter), here we have a (quasi free) mesons gas but with an effective chemical potential which contains the self-consistent interaction of the meson fields.

The numerical evaluation of the above thermodynamical quantities can be performed if the meson-nucleon, meson- $\Delta$ and meson-hyperon coupling constants are known. Concerning the 
meson-nucleon coupling constants they are determined to reproduce properties of equilibrium nuclear matter such as the saturation densities, the binding energy, the symmetric energy coefficient, the compression modulus and the effective Dirac mass at saturation. The set marked GM3 is from Ref. [3]. The implementation of hyperon degrees of freedom comes from determination of the corresponding meson-hyperon coupling constants that have been fitted to hypernuclear properties [5]. We refer to [4] for a detailed discussion on the dependence of the EOS from different $\Delta$-couplings).

In Fig. 1, we report the results of various particle-antiparticle ratios and $K^{+} / \pi^{+}$ratio as a function of the $\bar{p} / p$ ratio for different values of temperature. We can observe good agreement with the results obtained in the framework of statistical-thermal models and with experimental SPS and RHIC data [6].

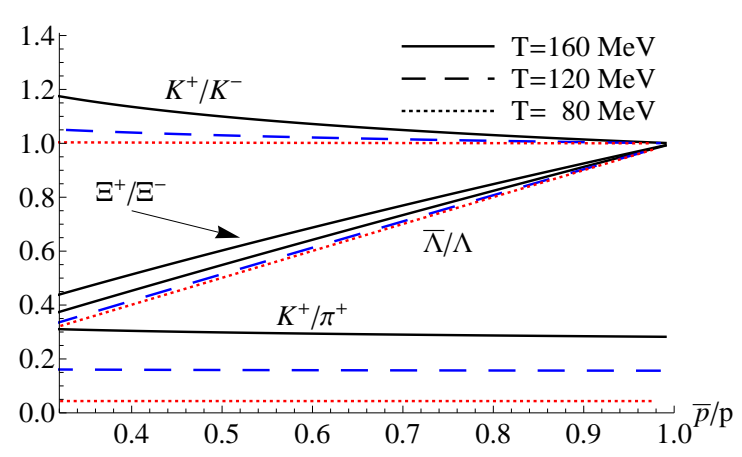

Figure 1: Particle-antiparticle and $K^{+} / \pi^{+}$ratios as a function of the $\bar{p} / p$ ratio for different temperatures. The $\Delta$ coupling ratios are fixed to $r_{s}=r_{v}=1$. The ratios of $\Xi^{+} / \Xi^{-}$at $T=80,120 \mathrm{MeV}$ are not reported because they are very strictly to the $\bar{\Lambda} / \Lambda$ ones.

In conclusion, we have studied an effective relativistic mean field model with the inclusion of the full octet of baryons, the $\Delta$-isobars and the lightest pseudoscalar and vector mesons, by requiring, in the range of finite density and temperature, the global conservation of baryon number, electric charge fraction and zero net strangeness. The meson degrees of freedom have been incorporated in the EOS as a quasi-particle Bose gas with an effective meson chemical potential $\mu^{*}$ expressed in terms of meson fields, responsible for the self-consistent mean field interaction. The introduced effective EOS is principally devoted to a regime of finite and intermediate values of baryon density and temperature reachable in the future compressed baryonic matter experiments.

\section{References}

[1] B.D. Serot, J.D. Walecka, Adv. Nucl. Phys. 16 (1986) 1.

[2] Z. Li, G. Mao, Y. Zhuo, W. Greiner, Phys. Rev. C 56 (1997) 1570.

[3] N.K. Glendenning, S.A. Moszkowski, Phys. Rev. Lett. 67, 2414 (1991).

[4] A. Lavagno, Phys. Rev. C 81 (2010) 044909.

[5] J. Schaffner-Bielich, A. Gal, Phys. Rev. C 62 (2000) 034311.

[6] B.I. Abelev et al. (STAR Collab.), Phys. Rev. C 79, 034909 (2009) and reference therein. 